\title{
Does really snacking positively reduce abdominal obesity?
}

\begin{abstract}
The prevalence of chronic diseases has increased worldwide in recent decades, focusing mainly on Obesity which became a major health problem for many age groups. Many biological and environmental factors have contributed to the current epidemic of obesity.

Speaking about obesity, snacking is one of the factors that have been understudied in relation to it. Studies were conducted to reveal whether snacking tend to increase weight or would help in weight reduction, since it is common in children and adolescents.

I strongly agree with many studies stating that snacking is inversely associated with overweight and abdominal obesity in both children and adolescents but the findings that relate snacking patterns to overweight are inconsistent, which makes them difficult to interpret. Many studies are needed to fully understand if snacking plays a role in childhood and adolescent's obesity.
\end{abstract}

Keywords: snacking, weight reduction, main meals, vegetables, fruits, obesity
Volume 8 Issue $3-2018$

\author{
Sara Rami El Zeitounie \\ Clinical Dietitian-Nutritionist, Saudi Arabia
}

Correspondence: Sara Rami El Zeitounie, Clinical Dietitian, Diet watchers, Riyadh, Saudi Arabia, Tel 009665078I8523, Email Sara.rami.93@gmail.com

Received: April 26, 2018 | Published: May 07, 2018

\section{Opinion}

Many former diet programs focused mainly on "three main meals" a day which made snacks of less importance compared to the main meals and led people believe that reducing food intake would aid in weight loss and started inventing strict diet programs that are actually not reaching the needed calories/day. This may cause many side effects including headaches, nausea, anemia as well as other life threating conditions.

Recent studies started revealing the benefits of snacking between meals and how effectively it can reduce weight and control appetite.

Snacking may be also an important strategy for meeting energy needs for increased physical activity and for meeting recommended intakes of short full nutrients that cannot be met with the consumption of only three meals/day. However, choice of snack is extremely important as choosing an unhealthy option might have an adverse effect on weight and health.

One study was made to clarify whether snacking is directly or inversely proportional with abdominal obesity and two important factors were taken into consideration:

\section{i. Snacking frequency.}

ii. Percentage of total daily energy intake contributed from snacks.

It revealed that: consuming two or more snacks/ day "increasing snack frequency and with increased percentage of energy from snacks" is associated with reduced risks of obesity especially abdominal obesity. This clearly showed that including snacks throughout the day is more successful at managing or even reducing weight and is associated with improved diet quality and increased intake of healthy options as: fruits, vegetables and whole grains which could promote satiety and reduce risks for obesity. ${ }^{1}$
Snacking is also associated with increased vigorous physical activity due to energy it provides before or after workout. It is possible that there could be an adverse association between snacking and weight gain if snackers exercise more than non-snackers because exercise is known to promote weight loss. Other benefits of snacking include prevention of hunger, as it provides energy and fiber that are both needed to help control appetite. It also tends to boost nutrient intakes that can't be fully provided with three main meals.

Eating frequently has the potential to positively affect metabolic parameters other than weight and body fat; these parameters include Cholesterol and Triglyceride levels as well as improved blood lipid and sugar. In addition, many studies have shown that "snack food" compared with food consumed at meals, are actually lower in fat (9 $\mathrm{kcal} / \mathrm{g}$ ) and higher in carbohydrates $(4 \mathrm{kcal} / \mathrm{g})$; therefore, individuals who snack regularly may be more successful at managing or even reducing weight by replacement of fat with healthy carbohydrates.

Snack may boost diet quality or lead to adverse effects as excess intake of solid fats, added sugars, and sodium and of course this all depends on "type of snack" chosen. Healthy options are unlimited, focusing mainly on high fiber, low sodium, low sugar as well as low fat, which include: fruits, vegetables, high fiber products as whole grains. $^{2}$

Being worried about weight gain and counting each and every calorie you include in your diet regimen is not something wrong, but always put into consideration that one of the most important factors for weight loss and increased metabolic rate is "Snacking". A wellbalanced diet with at least 4-5 meals/day is the key to a healthy weight management or even weight loss. Because all you need are delicious and healthy snacks flavoring your day! 


\section{Acknowledgements}

None.

\section{Conflict of interest}

Author declares no conflict of interest.

\section{References}

1. Debra R Keast, Theresa A Nicklas, Carol EO’Neil. Snacking is associated with reduced risk of overweight and reduced abdominal obesity in adolescents: National Health and Nutrition Examination Survey (NHANES). Am J Clin Nutr. 2010;92(2):428-435.

2. Food and Nutrition. Org. 